\title{
INDECOMPOSABLE DIVISION ALGEBRAS
}

\author{
B. A. SETHURAMAN
}

(Communicated by Maurice Auslander)

\begin{abstract}
We present a direct construction of indecomposable division algebras of all indices $p^{n}$ and exponents $p^{m} \quad(n \geq m \geq 2)$ that does not rely on generic methods.
\end{abstract}

\section{INTRODUCTION}

All division algebras will be finite dimensional over their centers. If $D$ is a division algebra with center $F$, then $D$ is said to admit a nontrivial decomposition if $D \cong D_{1} \otimes_{F} D_{2}$, where $1<\operatorname{dim}_{F}\left(D_{i}\right)<\operatorname{dim}_{F}(D)(i=1,2)$. If $D$ does not admit any nontrivial decompositions, $D$ is said to be indecomposable.

Given a division algebra $D$ with center $F$, the index of $D$ (ind $(D)$ ) is defined to be the square root of $\operatorname{dim}_{F}(D)$, and the exponent of $D(\exp (D))$ is defined to be the least integer $k$ such that $D \otimes_{F} \cdots \otimes_{F} D$ ( $k$ times) is isomorphic to a matrix ring over $F$. It is well known that $D$ is indecomposable only if ind $(D)$ is a power of a prime. Further, if $D$ has index and exponent $p^{n}$ for some prime $p$, then $D$ is necessarily indecomposable.

A natural question, then, is the following: Given an arbitrary prime $p$, do there exist indecomposable division algebras of all indices $p^{n}$ and exponents $p^{m}, n>m \geq 1$ ? This question (in its full generality) was first addressed by Saltman [S, Theorem 2.6], who showed that for $m \geq\{n / 2\}+1$ (where $\{n / 2\}$ is the least integer $\geq n / 2)$, the universal division algebras $U D\left(p^{n}, p^{m}\right)$ of index $p^{n}$ and exponent $p^{m}$ are indecomposable. Recently, using results derived from the geometry of Brauer-Severi varieties, Schofield and van den Bergh [SvdB, Theorem 2.2] showed that the algebras $U D\left(p^{n}, p^{m}\right)$ are indeed indecomposable for all $n$ and $m$ such that $n \geq m \geq 2$.

In this paper we show that indecomposable division algebra of all indices $p^{n}$ and exponents $p^{m}(n \geq m \geq 2)$ can be constructed directly from cyclic algebras. Our construction requires the ground field to contain enough roots of unity, but it does not rely on difficult generic methods. We then use this technique to obtain a concrete family of indecomposable division algebras whose centers are rational function fields.

Received by the editors October 12, 1990.

1980 Mathematics Subject Classification (1985 Revision). Primary 16A39.

Key words and phrases. Division algebras, indecomposability, cyclic algebras, symbol algebras.

This work constitutes a part of the author's doctoral dissertation, and was supported in part by the N.S.F. 
It is worth remarking that other special cases of indecomposable division algebras exist in the literature, for instance, Amitsur, Rowen, and Tignol [ART, Theorem 5.1], index 8 and exponent 2, Tignol [T, Theorem 2], index $p^{2}$ and exponent $p$ for odd primes $p$, and Jacob and Wadsworth [JW1, Remark 5.6], index $p^{n}$ and exponent $p^{m}(n>m \geq\{n / 2\}+1)$. Also, Rowen [R, Theorem 5] describes a family of indecomposable algebras; however, his results depend on an absence of roots of unity. Finally, Jacob [J, Theorem 3.3] describes indecomposable algebras of index $p^{n}(n \geq 2, n \geq 3$ if $p=2)$ and exponent $p$; his examples remain the only known nongeneric examples of exponent $p$ (and index an arbitrary power of $p$ ).

\section{THE CONSTRUCTION}

First, some notation. Let $F$ be a field containing all primitive $p^{j}$ th roots of unity $(j=1,2, \ldots)$, and for each $j$, choose a primitive $p^{j}$ th root of unity $\omega_{j}$ such that $\omega_{j}^{p}=\omega_{j-1}\left(j=1,2, \ldots\right.$, and where $\omega_{0}$ is understood to be 1). Given $a, b$ in $F-\{0\}$, we write $(a, b)_{p^{n}}$ for the algebra generated by two symbols $i, j$ subject to $i^{p^{n}}=a, j^{p^{n}}=b, i j=\omega_{n} j i$, and refer to such an algebra as a symbol algebra. It is well known that such an algebra is central simple over $F$, of dimension $p^{2 n}$. Thus, if $A$ denotes this algebra, then $A$ is isomorphic to a matrix ring over some division ring $D$. We define the index of $A(\operatorname{ind}(A))$ to be the index of $D$. Also, we define $A^{1 / p}$ to be the algebra $(a, b)_{p^{n+1}}$. By a standard identity (e.g. [D, Chapter 11, Lemma 6]), $\left(A^{1 / p}\right)^{p} \sim A$ in the Brauer group of $F$ (where $\left(A^{1 / p}\right)^{p}$ is the tensor product of $A^{1 / p}$ with itself $p$ times).

Proposition 1. Let $F$ be a field containing a primitive $p^{n+1}$ th root of unity $(n \geq$ 1). Let $a, b \in F-\{0\}$, and let $A=(a, b)_{p^{n}}$. Assume that $A$ is a division algebra (so its index is $\left.p^{n}\right)$, and let $p^{m}(0<m \leq n)$ be its exponent. Then $A^{1 / p}$ is an indecomposable division algebra of index $p^{n+1}$ and exponent $p^{m+1}$.

Proof. Clearly, ind $\left(A^{1 / p}\right) \leq p^{n+1}$. On the other hand, since $\operatorname{ind}(A)$ is a power of $p$, by [A, p. 76, Lemma7] we have ind $\left(A^{1 / p}\right) \geq p \cdot \operatorname{ind}\left(\left(A^{1 / p}\right)^{p}\right)=p \cdot \operatorname{ind}(A)=$ $p^{n+1}$. Thus, ind $\left(A^{1 / p}\right)$ is exactly $p^{n+1}$. Since we now have $\operatorname{ind}\left(A^{1 / p}\right)=$ $p \cdot \operatorname{ind}\left(\left(A^{1 / p}\right)^{p}\right), A^{1 / p}$ must be indecomposable by Saltman's criterion [S, Lemma 3.2]. Finally, since $\exp (A)=p^{m}, \exp \left(A^{1 / p}\right)$ must be $p^{m+1}$.

The proposition above thus reduces the construction of indecomposable division algebras of index $p^{n}$ and exponent $p^{m} \quad(n \geq m \geq 2)$ to the construction of symbol algebras of index $p^{n}$ and exponent $p^{m} \quad(n \geq m \geq 1)$. One family of such algebras are the generic cyclic algebras described in [O, Propositions 2.1 and 2.2] (if one assumes that the ground field there contains enough roots of unity). We describe below a different construction that yields division algebras that are defined over rational function fields and are somewhat easy to compute with. These examples were inspired by the construction in [T, Theorem 1].

Since the problem of indecomposablity is trivial if $m=n$, we focus on the case where $m<n$. Let $F_{0}$ be a field containing the primitive $p^{j}$ th roots of unity $\omega_{j}(j=1, \ldots, n+1)$ described above. Let $k=n-m$ (so $k \geq 1$ by assumption), and let $F=F_{0}\left(x_{0}, x_{1}, \ldots, x_{k}, y\right)$ be the rational function field 
in the $k+2$ indeterminates $x_{0}, x_{1}, \ldots, x_{k}, y$. We define $R_{n, m}$ by

$$
\begin{aligned}
R_{n, m}= & \left(x_{0}, x_{0}^{p^{k}}-y\right)_{p^{m}} \otimes_{F}\left(x_{1}, x_{1}^{p^{k-1}}-y\right)_{p} \otimes_{F}\left(x_{2}, x_{2}^{p^{k-2}}-y\right)_{p} \otimes_{F} \\
& \cdots \otimes_{F}\left(x_{k-1}, x_{k-1}^{p}-y\right)_{p} \otimes_{F}\left(x_{k}, x_{k}-y\right)_{p} .
\end{aligned}
$$

Proposition 2. $R_{n, m}$ is a division algebra of index $p^{n}$ and exponent $p^{m}$, and is isomorphic to $(y, a)_{p^{n}}$ for some $a$ in $F$.

Proof. For $j=0,1, \ldots, k$, let $E_{j}$ be the field $F_{0}\left(x_{0}, x_{1}, \ldots, x_{j}, y\right)$, and let $A_{j}$ be the central simple algebra over $E_{j}$ defined by

$A_{j}=\left(x_{0}, x_{0}^{p^{k}}-y^{p^{k-j}}\right)_{p^{m}} \otimes_{E_{j}}\left(x_{1}, x_{1}^{p^{k-1}}-y^{p^{k-j}}\right)_{p} \otimes_{E_{j}} \cdots \otimes_{E_{j}}\left(x_{j}, x_{j}^{p^{k-j}}-y^{p^{k-j}}\right)_{p}$

(so that $E_{k}$ is just $F$ and $A_{k}$ is just $R_{n, m}$ ). We will first show inductively that $A_{j}$ has index $p^{m+j}$, from which it will follow that $R_{n, m}$ has index $p^{n}$. We will use valuation-theoretic results from [JW2] to establish this.

Assume $j=0$. Consider $A_{0}=\left(x_{0}, x_{0}^{p^{k}}-y^{p^{k}}\right)_{p^{m}}$. Recall that $\left(x_{0}^{p^{k}}-y^{p^{k}}\right)$ factors completely into linear factors of the form $\left(x_{0}-\omega_{k}^{i} y\right)(i=0,1, \ldots$, $\left.p^{k}-1\right)$. With respect to the $\left(x_{0}-y\right)$-adic valuation on $E_{0}$ (i.e., the discrete valuation obtained by localizing $F_{0}\left[x_{0}, y\right]$ at the height 1 prime ideal generated by $\left.\left(x_{0}-y\right)\right)$ the field $E_{0}\left(x_{0}^{1 / p^{m}}\right)$ is unramified over $E_{0}$. In particular, the valuation extends uniquely from $E_{0}$ to $E_{0}\left(x_{0}^{1 / p^{m}}\right)$ and the value group does not grow. Hence the value of the norm from $E_{0}\left(x_{0}^{1 / p^{m}}\right)$ to $E_{0}$ of any nonzero element in $E_{0}\left(x_{0}^{1 / p^{m}}\right)$ is a multiple of $p^{m}$. Since $\left(x_{0}^{p^{k}}-y^{p^{k}}\right)$ has value 1 , it follows that $\left(x_{0}^{p^{k}}-y^{p^{k}}\right)^{l}$ cannot be the norm of an element in $E_{0}\left(x_{0}^{1 / p^{m}}\right)$ for $0<l<p^{m}$. Hence $A_{0}$ has exponent, and consequently index, $p^{m}$.

Now assume that $0<j \leq k$ and that $A_{j-1}$ has been shown to have index $p^{m+j-1}$. Let $E_{j, h}$ denote the henselization of $E_{j}$ with respect to the $\left(x_{j}-y\right)$-adic valuation, and consider $A_{j, h}=A_{j} \otimes_{E_{j}} E_{j, h}$. Notice that the factor $\left(x_{j}, x_{j}^{p^{k-j}}-y^{p^{k-j}}\right)_{p} \otimes_{E_{j}} E_{j, h}(=N$, say) has the same form as [JW2, Example 4.3]. In particular, it is a nicely semiramified division algebra, in the terminology of [JW2, §4]. (Of course, $N$ can also be seen to be a division algebra by an argument analagous to that used for $A_{0}$.) Recalling that the residues of $x_{j}$ and $y$ are equal with respect to this valuation, we find that the residue of $E_{j, h}$ (which equals the residue of $E_{j}$ ) is just $E_{j-1}$ and that the residue $\bar{N}$ of $N$ is $E_{j-1}\left(y^{1 / p}\right)$. If we put $z=y^{1 / p}$, then $\bar{N}$ is just $F_{0}\left(x_{0}, x_{1}, \ldots, x_{j-1}, z\right)$, a rational function field in $j+1$ variables. Now observe that the elements $x_{i}$ and $x_{i}^{p^{k-i}}-y^{p^{k-j}}(i=0,1, \ldots, j-1)$ are all units with respect to the $\left(x_{j}-y\right)$-adic valuation. Let $I$ denote the division algebra similar to $\left(x_{0}, x_{0}^{p^{k}}-y^{p^{k-j}}\right)_{p^{m}} \otimes_{E_{j, h}}\left(x_{1}, x_{1}^{p^{k-1}}-y^{p^{k-j}}\right)_{p} \otimes_{E_{j, h}} \cdots \otimes_{E_{j, h}}$ $\left(x_{j-1}, x_{j-1}^{p^{k-(j-1)}}-y^{p^{k-j}}\right)_{p}$ in the Brauer group of $E_{j, h}$. The $\left(x_{j}-y\right)$-adic valuation extends to $I$ since $E_{j, h}$ is henselian. It follows from [JW2, Example 2.4(i) and Proposition 2.5] that $I$ is inertial over $E_{j, h}$, and if $\bar{I}$ denotes the residue of $I$, that $\bar{I} \otimes_{E_{j-1}} \bar{N}$ is similar to the algebra $\left(x_{0}, x_{0}^{p^{k}}-z^{p^{k-(j-1)}}\right)_{p^{m}}$ $\otimes_{\bar{N}}\left(x_{1}, x_{1}^{p^{k-1}}-z^{p^{k-(j-1)}}\right)_{p} \otimes_{\bar{N}} \cdots \otimes_{\bar{N}}\left(x_{j-1}, x_{j-1}^{p^{k-(j-1)}}-z^{p^{k-(j-1)}}\right)_{p}$ in the 
Brauer group of $\bar{N}$. This has the same form as $A_{j-1}$ (but defined, instead, over $F_{0}\left(x_{0}, x_{1}, \ldots, x_{j-1}, z\right)$ ), so, by induction, it has index $p^{m+j-1}$. Hence, by [JW2, Theorem 5.15], ind $\left(A_{j, h}\right)=\operatorname{ind}(N) \cdot \operatorname{ind}\left(\bar{I} \otimes_{E_{j-1}} \bar{N}\right)=p \cdot p^{m+j-1}$ $=p^{m+j}$. Thus, ind $\left(A_{j}\right) \geq \operatorname{ind}\left(A_{j, h}\right) \geq p^{m+j} . \operatorname{But} \operatorname{ind}\left(A_{j}\right) \leq \sqrt{\operatorname{dim}_{E_{j}}\left(A_{j}\right)}=$ $p^{m+j}$. Hence, $A_{j}$ has index exactly $p^{m+j}$.

To prove that $R_{n, m}$ has exponent $p^{m}$, note that, if $m>1, R_{n, m}^{p} \sim$ $\left(x_{0}, x_{0}^{p^{k}}-y\right)_{p^{m-1}}$. By the same argument used for $A_{0}$ above, but applied instead to the $\left(x_{0}^{p^{k}}-y\right)$-adic valuation, it can be seen that $R_{n, m}^{p}$ has exponent $p^{m-1}$. Hence $R_{n, m}$ must have exponent $p^{m}$.

Finally, we establish the required isomorphism. Recall the identity $(a, a-b)_{p^{\prime}} \sim(b,(a-b) / a)_{p^{\prime}} \otimes_{F}(a,-1)_{p^{l}}, l=1,2, \ldots$, valid for any $a, b \in F-\{0\}$ with $a-b \neq 0$ [D, Chapter 11, Lemma 11]. Notice that -1 is a $p^{l}$ th power for $l=1, \ldots, n$, by the assumption about the roots of unity in $F$. Using this, along with standard properties of symbol algebras (e.g. [D, Chapter 11, Lemmas 4 and 6]), we get for $j=1, \ldots, k$ that

$$
\left(x_{j}, x_{j}^{p^{k-j}}-y\right)_{p} \sim\left(x_{j}^{p^{k-j}}, x_{j}^{p^{k-j}}-y\right)_{p^{k-j+1}} \sim\left(y, \frac{x_{j}^{p^{k-j}}-y}{x_{j}^{p^{k-j}}}\right)_{k-j+1} \sim\left(y, a_{j}\right)_{p^{n}},
$$

where we have written $a_{j}$ for

$$
\left(\frac{x_{j}^{p^{k-j}}-y}{x_{j}^{p^{k-j}}}\right)^{p^{n-(k-j+1)}}
$$

Similarly, $\left(x_{0}, x_{0}^{p^{k}}-y\right)_{p^{m}} \sim\left(y, a_{0}\right)_{p^{n}}$, where $a_{0}=\left(x_{0}^{p^{k}}-y\right) / x_{0}^{p^{k}}$. Writing $a$ for the product of $a_{0}$ and the $a_{j}$ 's $(j=1, \ldots, k)$, and noting that the index of $R_{n, m}$ is $p^{n}$, we get that $R_{n, m}$ is indeed isomorphic to $(y, a)_{p^{n}}$.

Theorem. The algebras $R_{n, m}^{1 / p}\left(=(y, a)_{p^{n+1}}\right)$ are indecomposable division algebras of index $p^{n+1}$ and exponent $p^{m+1} \quad(n>m \geq 1)$.

Proof. This is immediate from Propositions 1 and 2.

\section{REFERENCES}

[A] A. A. Albert, Structure of algebras, Amer. Math. Soc. Colloq. Pub. vol. 24, Providence, RI, 1961.

[ART] S. A. Amitsur, L. H. Rowen, and J.-P. Tignol, Division algebras of degree 4 and 8 with involution, Israel J. Math. 33 (1979), 133-148.

[D] P. K. Draxl, Skew fields, London Math. Soc. Lecture Note Ser., Vol. 81, Cambridge Univ. Press, Cambridge, 1983.

[J] B. Jacob, Indecomposible division algebras of prime exponent, J. Reine Angew. Math. (to appear).

[JW1] B. Jacob and A. Wadsworth, A new construction of noncrossed product algebras, Trans. Amer. Math. Soc. 293 (1986), 693-721.

[JW2] _ Division algebras over henselian fields, J. Algebra 128 (1990), 126-179.

[O] C. Ousmane, Algèbres cycliques génériques, Bull. Soc. Math. Belg. Sér. B 40 (1988), 43-59. 
[R] L. H. Rowen, Cyclic division algebras, Israel J. Math. 41 (1982), 213-234.

[S] D. Saltman, Indecomposable division algebras, Comm. Algebra 7 (1979), 791-817.

[SvdB] A. Schofield and M. van den Bergh, The index of a Brauer class on a Brauer-Severi variety, Trans. Amer. Math. Soc. (to appear).

[T] J.-P. Tignol, Algèbres indécomposables d'exposant premier, Adv. in Math. 65 (1987), 205228.

Department of Mathematics, University of California at San Diego, la Jolla, CaliFORNIA 92093 